\title{
Mathematics of Dispersive Water Waves
}

\author{
B. A. Kupershmidt
}

The University of Tennessee Space Institute, Tullahoma, TN 37388, USA, and

Center for Nonlinear Studies, Los Alamos National Laboratory, Los Alamos, NM 87545, USA

\begin{abstract}
A commuting hierarchy of dispersive water wave equations makes a three-Hamiltonian system which belongs to a general class of nonstandard integrable systems whose theory is developed. The modified water wave hierarchy is a bi-Hamiltonian system; its modification bifurcates. The water wave hierarchy, and the hierarchies of the Korteweg-de Vries and the modified Korteweg-de Vries equations, as well as the classical Miura map, are given new representations through various specializations of nonstandard systems.
\end{abstract}

\section{Introduction}

The current theory of integrable systems grew out of the analysis of the Kortewegde Vries equation. Another, even more remarkable water wave equation, is the subject of this paper.

The classical dispersiveless long wave equations

$$
u_{t}+u u_{x}+h_{x}=0, h_{t}+(u h)_{x}=0, u=u(x, t), h=h(x, t),
$$

have a number of dispersive generalizations (see, e.g., review [1]). In this paper we shall be concerned with the following version

$$
\left\{\begin{array}{l}
u_{t}=\left(u^{2} / 2+h+\beta u_{x}\right)_{x} \\
h_{t}=\left(u h+\alpha u_{x x}-\beta h_{x}\right)_{x}
\end{array}\right.
$$

here $\alpha$ and $\beta$ are arbitrary constants, and the sign of $t$ is changed to make forthcoming formulae more natural. The invertible change of variables: $u=\bar{u}$, $h=\bar{h}+\gamma \bar{u}_{x}$, turns (1.2) into

$$
\left\{\begin{array}{l}
\bar{u}_{t}=\left(\bar{u}^{2} / 2+\bar{h}+\mu \bar{u}_{x}\right)_{x}, \\
\bar{h}_{t}=\left(\bar{u} \bar{h}-\mu \bar{h}_{x}\right)_{x}, \quad \mu=\gamma+\beta= \pm \sqrt{\alpha+\beta^{2}} .
\end{array}\right.
$$

If $\mu$ does not vanish, it can be made into an arbitrary constant, say $\mu= \pm 1 / 2$, by rescaling $x$ and $t$. For $\alpha=1 / 3, \beta=0$, the system (1.2) was derived by Broer [1] who called it "The oldest, simplest and most widely known set of equations...", 
“...which are the Boussinesq equations proper." The same system (1.2), for $\beta=0$ and in terms of the potential $\varphi: u=\varphi_{x}$, was derived by Kaup [2] who also found its multi-soliton solutions and the following scattering problem: $\psi_{x x}+\left(\lambda^{2}+\lambda Q+R\right) \psi=0$, where $Q$ and $R$ are obtained by an invertible change of variables from $u$ and $h$. Later Matveev and Yavor [3] found algebro-geometrically a large class of almost periodic solutions "... of some hydrodynamical equations derived recently by D. Kaup."

We shall see that the system (1.3) is connected with a large variety of ideas in the theory of integrable systems, which are important in their own right. Briefly, this system is the richest integrable system known to date. In this paper we establish the basic properties of the system (1.3) and the corresponding hierarchy by making use of the theory of nonstandard integrable systems [Eq. (2.3) below] developed in Sect. 2; the most important results are:

Theorem 2.0. Consider the hierarchy

$$
\begin{gathered}
L_{t}=\left[\left(\left(P^{\dagger}\right)_{\geqq 1}\right)^{\dagger}, L\right]=\left[-\left(\left(P^{\dagger}\right)_{\leqq 0}\right)^{\dagger}, L\right], \\
L=\xi+u+h \xi^{-1}, \\
P \in Z(L),
\end{gathered}
$$

where $\xi=d / d x ; Z(L)$ is the centralizer of $L$ in the ring of pseudo-differential operators $C\left(\left(\xi^{-1}\right)\right)([4,5]), C=C_{u, h}=\mathbb{C}\left[u^{(i)}, h^{(i)}\right]$ is the corresponding differential algebra; " $\dagger$ " stands for the "adjoint"; for an element $Q \in C\left(\left(\xi^{-1}\right)\right), Q=\sum_{i<\infty} q_{i} \xi^{i}$, we denote $\operatorname{Res} Q=q_{-1}, Q_{\geqq k}=\sum_{i \geqq k} q_{i} \xi^{i}$, and analogously for $Q_{>k}, Q_{<k}, Q_{\leqq k}$. Then:

(i) The hierarchy (1.4) has a common infinite set of conservation laws $H_{m}=\frac{1}{m} \operatorname{Res} L^{m}$; (ii) All these flows commute.

Since the centralizer $Z(L)$ of $L$ is a linear combination of $\left\{L^{n} \mid n \in \mathbb{Z}\right\}$, we can take $P=\frac{1}{2} L^{2}$ in (1.4).

The resulting system is readily found to be

$$
\left\{\begin{array}{l}
u_{t}=\partial\left(u^{2}+2 h-u_{x}\right) / 2, \quad \partial=\partial / \partial x, \\
h_{t}=\partial\left(2 u h+h_{x}\right) / 2
\end{array}\right.
$$

that is, (1.3) with $\mu=-1 / 2$.

Theorem 3.0. The hierarchy (1.4) is a three-Hamiltonian system: (i) for $P=L^{m}$ it can be written in the form

$$
\left(\begin{array}{l}
u \\
h
\end{array}\right)_{t}=B^{\mathrm{I}} \delta H_{m+1}=B^{\mathrm{II}} \delta H_{m}=B^{\mathrm{III}} \delta H_{m-1},
$$

where $\delta H=\left(\begin{array}{l}\delta H / \delta u \\ \delta H / \delta h\end{array}\right)$ is the vector of variational derivatives, and

$$
\begin{gathered}
B^{\mathrm{I}}=\left(\begin{array}{ll}
0 & \partial \\
\partial & 0
\end{array}\right), \\
B^{\mathrm{II}}=\left(\begin{array}{cc}
2 \partial & \partial u-\partial^{2} \\
u \partial+\partial^{2} & h \partial+\partial h
\end{array}\right),
\end{gathered}
$$




$$
B^{\mathrm{III}}=\left(\begin{array}{cc}
2(u \partial+\partial u) & 2(h \partial+\partial h)+\partial(u-\partial)^{2} \\
2(h \partial+\partial h)+(u+\partial)^{2} \partial & (u+\partial)(h \partial+\partial h)+(h \partial+\partial h)(u-\partial)
\end{array}\right) .
$$

(ii) All matrices $B^{\mathrm{I}}, B^{\mathrm{II}}, B^{\mathrm{III}}$ are Hamiltonian.

Theorem 4.0. (i) The system (1.4) with $P=L^{m}$, and for $-u$ instead of $u$, has also the following Lax representation

$$
\mathscr{L}_{t}=\left[\mathscr{P}_{\geqq 0}, \mathscr{L}\right]=\left[-\mathscr{P}_{<0}, \mathscr{L}\right],
$$

where $\mathscr{P}=\mathscr{L}^{m}$ and

$$
\mathscr{L}=\xi+\sum_{i \geqq 0} \xi^{-i-1} h Q_{i}(u)
$$

where

$$
Q_{i}(u)=(\partial+u)^{i}(1)
$$

(ii) The conservation laws $H_{m}=\frac{1}{m} \operatorname{Res} L^{m}$ and $\mathscr{H}_{m}=\frac{1}{m} \operatorname{Res} \mathscr{L}^{m}$ coincide.

Theorem 5.0. The Korteweg-de Vries (KdV) hierarchy

$$
\ell_{t}=\left[\tilde{P}_{\geqq 0}, \ell\right]=\left[-\tilde{P}_{<0}, \ell\right], \ell=\xi^{2}+h, \tilde{P}=\left(\ell^{1 / 2}\right)^{2 m+1}, \quad m \in \mathbb{Z}_{+},
$$

can be imbedded into the hierarchy (1.4) when $u$ vanishes and $P=L^{2 m+1}$. In other words, set

$$
\bar{L}=\xi+h \xi^{-1}
$$

and take $\bar{P}=\bar{L}^{2 m+1}$. Then: (i) The system $\bar{L}_{t}=\left[\left(\left(\bar{P}^{\dagger}\right)_{\geqq 1}\right)^{\dagger}, \bar{L}\right]$ is the $m^{\text {th }} \mathrm{KdV}$ flow; (ii) The Hamiltonian structures $B^{\mathrm{I}}(1.9)$ and $B^{\mathrm{III}}(1.11)$ can be properly restricted into the first and the second Hamiltonian structure, respectively, of the $\mathrm{KdV}$ hierarchy.

Theorems 2.0-5.0 are proved below in Sects. 2-5 respectively, based on a theory of nonstandard integrable systems developed in Sect. 2. Details, explanations, and generalizations can be found in the course of the paper. Elementary facts about differential Lax equations are assumed to be known (see [4, 5]). Here I explain the method, devised in [6] for discrete Lax equations, used to prove Theorem 3.0. As an example, let us take the $\mathrm{KdV}$ hierarchy $\ell_{t}=\left[\ell, \widetilde{P}_{<0}\right]$, with $\ell=\xi^{2}+h, \tilde{P}=\left(\ell^{1 / 2}\right)^{2 m+1}$. Set $H_{m}=m^{-1} \operatorname{Res}\left(\ell^{1 / 2}\right)^{m},\left(\ell^{1 / 2}\right)^{m}=\sum_{s} p_{s}(m) \xi^{s}$. Then the $m^{\text {th }} \mathrm{KdV}$ equation becomes $h_{t}=2 \partial p_{-1}(2 m+1)$. On the other hand, recall that the basic formula of the Residue calculus in modules of differential forms over rings of pseudo-differential operators [5] is

$$
\frac{1}{m+n} d \operatorname{Res} X^{m+n} \sim \frac{1}{n} \operatorname{Res}\left(X^{m} d X^{n}\right) \sim \frac{1}{n} \operatorname{Res}\left(d X^{n} \cdot X^{m}\right), \quad m, n \in \mathbb{Z},
$$

for any pseudo-differential operator $X \in C\left(\left(\xi^{-1}\right)\right)$, where $d$ is the differential, and $a \sim b$ means: $(a-b) \in \operatorname{Im} \partial$. Taking $X=\ell^{1 / 2}, n=2$, we obtain $\frac{1}{m+2} d \operatorname{Res}\left(\ell^{1 / 2}\right)^{m+2}$ $=d H_{m+2} \sim \frac{1}{2} \operatorname{Res}\left(d \ell \circ\left(\ell^{1 / 2}\right)^{m}\right)=\frac{1}{2} \operatorname{Res}\left(d h \sum p_{s}(m) \xi^{s}\right)=\frac{1}{2} p_{-1}(m) d h$. Therefore

$$
p_{-1}(m)=2 \frac{\delta H_{m+2}}{\delta h},
$$


and substituting this into $h_{t}=2 \partial p_{-1}(2 m+1)$, we obtain the first Hamiltonian representation

$$
h_{t}=2 \partial p_{-1}(2 m+1)=4 \partial \frac{\delta H_{2 m+3}}{\delta h} .
$$

To obtain the second Hamiltonian form we need to re-express $\partial p_{-1}(2 m+1)$ through $p_{-1}(2 m-1)$. For this, we write

$$
\left(\ell^{1 / 2}\right)^{m+2}=\left(\ell^{1 / 2}\right)^{m} \ell=\ell\left(\ell^{1 / 2}\right)^{m},
$$

and pick out the $\xi^{-1}$ - and $\xi^{-2}$-terms from both sides:

$$
\begin{gathered}
p_{-1}(m+2)=p_{-3}(m)+p_{-1}(m) h, \\
p_{-1}(m+2)=p_{-3}(m)+2 \partial p_{-2}(m)+\partial^{2} p_{-1}(m)+h p_{-1}(m), \\
p_{-2}(m+2)=p_{-4}(m)+p_{-2}(m) h-p_{-1}(m) \partial h, \\
p_{-2}(m+2)=p_{-4}(m)+2 \partial p_{-3}(m)+\partial^{2} p_{-2}(m)+h p_{-2}(m) .
\end{gathered}
$$

Comparing (1.19) with (1.20) we obtain

$$
p_{-2}(m)=-\frac{1}{2} \partial p_{-1}(m)
$$

[recall that $r k p_{s}(m)=m-s$ with $\left.r k h^{(j)}=j+2\right]$. Similarly, comparing (1.21) with (1.22), we obtain

$$
\begin{aligned}
2 \partial p_{-3}(m) & =-p_{-1}(m) \partial h-\partial^{2} p_{-2}(m)=[\text { by }(1.23)] \\
& =-p_{-1}(m) \partial h+\frac{1}{2} \partial^{3} p_{-1}(m) .
\end{aligned}
$$

Substituting this into (1.19) we get

$$
\begin{aligned}
2 \partial p_{-1}(m+2) & =-p_{-1}(m) \partial h+\frac{1}{2} \partial^{3} p_{-1}(m)+2 \partial\left(p_{-1}(m) h\right) \\
& =\left(\frac{1}{2} \partial^{3}+h \partial+\partial h\right) p_{-1}(m),
\end{aligned}
$$

thus

$$
h_{t}=2 \partial p_{-1}(2 m+1)=4 \partial \frac{\partial H_{2 m+3}}{\delta h}=\left[\partial^{3}+2(h \partial+\partial h)\right] \frac{\delta H_{2 m+1}}{\delta h},
$$

and this is the second Hamiltonian form of the KdV hierarchy.

\section{Nonstandard Integrable Systems}

The strangely looking system (1.4) is a particular case of the following general setup. For the rest of the paper, $k=0,1$ or 2 . Let

or

$$
L=\sum_{-k}^{n} u_{i} \xi^{i}
$$

$$
L=\sum_{-\infty}^{n} u_{i} \xi^{i}
$$

with $n \geqq 1$ and the normalization conditions

$$
k=0: u_{n}=1, u_{n-1}=0 ; \quad k=1: u_{n}=1 .
$$


Denote $C=C_{u}=\mathbb{C}\left[u_{i}^{(j)}\right]$ the differential algebra generated by $u_{i}$ 's (see [5]). The centralizer $Z(L)$ of $L$ in $C\left(\left(\xi^{-1}\right)\right.$ ) is generated over $\mathbb{C}$ by $\left(L^{1 / n}\right)^{m}, m \in \mathbb{Z}$ (where we assume $u_{n}=\bar{u}_{n}^{n}$ for some fixed $\bar{u}_{n}$ when $k=2$ ). For $P \in Z(L)$, consider the following system,

$$
L_{t}=\left[\left(\left(P^{\dagger}\right)_{\geqq k}\right)^{\dagger}, L\right]=\left[-\left(\left(P^{\dagger}\right)_{<k}\right)^{\dagger}, L\right] .
$$

Since $\left(\left(P^{\dagger}\right)_{<k}\right)^{\dagger}$ is an operator of order $\leqq k-1$, the right-hand side of $(2.3)$ has order $\leqq n+k-2$, which guarantees the preservation of the normalization conditions (2.2). For $k=0$ we recover the standard differential Lax equations, $L_{t}=\left[P_{\geq 0}, L\right]$ $=\left[-P_{<0}, L\right]([4,5])$, since only for $k=0$ the projection $X \mapsto X_{<k}, X \in C\left(\left(\bar{\xi}^{-1}\right)\right)$, and taking the adjoint, $X \mapsto X^{\dagger}$, commute. For the case (2.1a) of finite $L$ and $k>0$, it remains to show that the right-hand side of $(2.3)$ belongs to $C\left(\left(\xi^{-1}\right)\right)_{\geq-k}$. Set $\left(P^{\dagger}\right)_{\geqq k}=Q \xi^{k}$ with some $Q \in C[\xi]=C\left(\left(\xi^{-1}\right)\right)_{\geqq 0}$. Then

$$
\begin{gathered}
{\left[\left(Q \xi^{k}\right)^{\dagger}, L\right]_{<0}=\left[\left(Q \xi^{k}\right)^{\dagger}, L_{<0}\right]_{<0}=\left[(-\xi)^{k} Q^{\dagger}, \sum_{i=-k}^{-1} u_{i} \xi^{i}\right]_{<0}} \\
=\left[\sum_{-k}^{-1}(-\xi)^{k} Q^{\dagger} u_{i} \xi^{i}-\sum_{-k}^{-1} u_{i} \xi^{i}(-\xi)^{k} Q^{\dagger}\right]_{<0}=\left[\sum_{-k}^{-1}(-\xi)^{k} Q^{\dagger} u_{i} \xi^{i}\right]_{<0} \in C\left(\left(\xi^{-1}\right)\right)_{\geqq-k} .
\end{gathered}
$$

Theorem 2.5. For fixed $L$, the evolution derivations of $C$ given by (2.3) for various $P \in Z(L)$, commute between themselves and have an infinite common set of conservation laws (=c.1's)

$$
H_{m}=\frac{1}{m} \operatorname{Res}\left(L^{1 / n}\right)^{m}, \quad m \in \mathbb{N} .
$$

Proof. Let $L$ be as in (2.1b) and denote by $I_{k}$ the differential ideal in $C$ generated by $\mathbb{C}\left[u_{i}^{(j)}\right]_{i<-k}$. Then from (2.3) and (2.4) we obtain

$$
\left(L_{<-k}\right)_{t}=\left[\left(\left(P^{\dagger}\right)_{\geqq k}\right)^{\dagger}, L\right]_{<-k}=\left[\left(\left(P^{\dagger}\right)_{\geqq k}\right)^{\dagger}, L_{<-k}\right]_{<-k},
$$

so that $\left(I_{k}\right)_{t} \subset I_{k}$. Therefore, the case (2.1a) is a specialization $C / I_{k}$ of the universal infinite case (2.1b), and we now restrict ourselves to this case only. Firstly, suppose $n=1$. Then from (2.3) we get $\left(L^{m}\right)_{t}=\left[\left(\left(P^{\dagger}\right)_{\geqq k}\right)^{\dagger}, L^{m}\right]$, and since Res $([],) \sim 0$, we obtain an infinity of c.l's $H_{m}$. From the existence of the Hamiltonian forms of the system (2.3) (derived below) it follows that all these c.l's are in involution; thus, all the flows commute. If now $n>1$, we let $\bar{L}=L^{1 / n}=\sum_{-\infty}^{1} \bar{u}_{i} \xi^{i}$. The map $L \rightarrow \bar{L}$ generates an invertible differential map (automorphism) $C_{\bar{u}}=\mathbb{C}\left[\bar{u}_{i}^{(j)}\right] \rightarrow C_{u}=\mathbb{C}\left[u_{i}^{(j)}\right]$ with respect to which $Z(L)$ and $Z(\bar{L})$ are "the same" too (isomorphic). Thus, each of Eq. (2.3) for $L$ and $\bar{L}$ implies the other. In particular, the derivations of $C_{u}$ commute since those of $C_{\bar{u}}$ do, as we found above.

Now we compute the (first) Hamiltonian form of the system (2.3). Set

$$
P=L^{m}=\sum_{s} \xi^{s} p_{s}(m) .
$$

From (1.16) with $X=L$, we obtain

$$
\begin{aligned}
d H_{m+1} & =\frac{1}{m+1} d \operatorname{Res} L^{m+1} \sim \operatorname{Res}\left(d L \circ L^{m}\right)=\operatorname{Res}\left(\sum_{i} d u_{i} \xi^{i} \sum_{s} \xi^{s} p_{s}(m)\right) \\
& =\sum d u_{i} p_{-i-1}(m),
\end{aligned}
$$


and thus

$$
p_{-i-1}(m)=\frac{\delta H_{m+1}}{\delta u_{i}}, 1 \geqq i>-\infty,
$$

where we temporarily abandon the normalization conditions (2.2) and treat $u_{1}$ and $u_{0}$ as legitimate variables; the advantages of such course of action will be seen later on in the case $k \neq 0$. We consider separately three cases: $k=0,1,2$.

0) $k=0$. Set $v_{i}=u_{-i-1}$, so that

$$
\begin{gathered}
L=\xi+\sum_{i \geqq 0} v_{i} \xi^{-i-1}, \\
p_{i}(m)=\frac{\delta H_{m+1}}{\delta v_{i}}, i \geqq 0 .
\end{gathered}
$$

Denote

$$
X_{i}=\frac{\delta H_{m+1}}{\delta v_{i}}
$$

Then

$$
\left(\left(P^{\dagger}\right)_{\geqq 0}\right)^{\dagger}=P_{\geqq 0}=\sum_{s \geqq 0} \xi^{s} X_{s},
$$

and

$$
\begin{gathered}
\partial_{t}\left(v_{i}\right)=\xi^{-i-1} \text {-coef. in } \\
{\left[\sum_{s \geqq 0} \xi^{s} X_{s}, \xi+\sum_{j \geqq 0} v_{j} \xi^{-j-1}\right]=\xi^{-i-1} \text {-coef. in }} \\
{\left[\sum_{s \geqq 0} \xi^{s} X_{s}, \sum_{j \geqq 0} v_{j} \xi^{-j-1}\right]=: \sum_{j} B_{i j}\left(X_{j}\right),}
\end{gathered}
$$

where $B_{i j}$ is a differential operator depending linearly upon $v$ 's. (We do not compute the matrix elements $B_{i j}$ at the moment since in a little while we will change our $L$.) For $B=\left(B_{i j}\right)$ to be Hamiltonian, the corresponding algebra, say $\mathfrak{b}$, must be a Lie algebra [6-8]. To compute multiplication in $\mathfrak{6}$, we notice that, setting

$$
X=\sum_{i \geqq 0} \xi^{i} X_{i}, Y=\sum_{j \geqq 0} \xi^{j} Y_{j}, v=\sum_{j \geqq 0} v_{j} \xi^{-j-1}
$$

we obtain

$$
\begin{aligned}
\bar{Y}^{t} B \bar{X}: & =\sum_{i, j} B_{i j}\left(X_{j}\right) \cdot Y_{i}=\xi^{-1} \text {-coef. in }([X, v] Y)=\operatorname{Res}([X, v] Y) \\
& \sim[\text { since } \operatorname{Res}(,) \text { is an invariant form }] \sim \operatorname{Res}(v[Y, X]) \\
& =\sum_{j} v_{j}\left\{\text { left } \xi^{j} \text {-coef. in }[Y, X]\right\}=\sum_{j} v_{j}[Y, X]_{j},
\end{aligned}
$$

where $\sum_{j} \xi^{j}[Y, X]_{j}=[Y, X]$. Thus $(\mathfrak{5}$ is the Lie algebra of differential operators, therefore the matrix $B=\left(B_{i j}\right)$, being the natural Hamiltonian matrix on the dual space $\mathfrak{5}^{*}$, is indeed Hamiltonian. However, the coordinates we have ended up with 
on $\mathfrak{G}$ are not convenient, since the differential operators are written in the left form $\sum \xi^{j} X_{j}$ instead of the usual right form. Obviously, the root of the problem is $L$ itself: as it is clear from the derivation of (2.8), we need to work with $L$ in the left form in order for $P$ to be naturally represented in the right form. The remedy, then, is clear: taking adjoint of $(2.3)$ we obtain $-L_{t}^{\dagger}=\left[\left(P^{\dagger}\right)_{\geqq k}, L^{\dagger}\right]$, with $P^{\dagger}=\left(L^{\dagger}\right)^{m}$. Thus, we can discard the form (2.3) and instead use the following form:

$$
\begin{gathered}
L_{t}=\left[P_{\geqq k}, L\right]=\left[-P_{<k}, L\right], \\
L=\sum_{-\infty}^{n} \xi^{i} u_{i}, \quad n=1,
\end{gathered}
$$
with $P=L^{m}, m \in \mathbb{Z}_{+}$. Setting $H=\frac{1}{m} \operatorname{Res} L^{m}, P=L^{m}=\sum_{s} p_{s}(m) \xi^{s}$, we again obtain
(2.8).

For $k=0$, repeating the derivation of (2.11) and (2.12), with $v_{s}=u_{-s-1}$ and $u_{1}=1, u_{0}=0$, we obtain

$$
\partial_{t}\left(v_{i}\right)=\operatorname{Res}\left(\xi^{i}\left[\sum_{s \geqq 0} X_{s} \xi^{s}, \sum_{j \geqq 0} \xi^{-j-1} v_{j}\right]\right)=\sum_{j \geqq 0} B_{i j}^{0}\left(X_{j}\right),
$$

and setting $X=\sum_{s \geqq 0} X_{s} \xi^{s}, Y=\sum_{i \geqq 0} Y_{i} \xi^{i}, v=\sum_{j \geqq 0} \xi^{-j-1} v_{j}$, we get

$$
\bar{Y}^{t} B^{0} \bar{X}=\sum_{i, j} Y_{i} B_{i j}^{0}\left(X_{j}\right)=\operatorname{Res}(Y[X, v]) \sim \operatorname{Res}([Y, X] v)=\sum_{j} v_{j}[Y, X]_{j},
$$

where $\sum_{j}[Y, X]_{j} \xi^{j}=[Y, X]$. Thus $B^{0}$ is the natural Hamiltonian matrix on the dual space to the Lie algebra of differential operators. In the proof of Theorem 4.0, we shall need the explicit form of the matrix elements of the matrix $B^{r}=\left(B_{i j}^{r}\right)$ associated to the dual space of the Lie algebra $\mathfrak{G}_{\geqq r}$ of (right) differential operators of order $\geqq r\left(\in \mathbb{Z}_{+}\right)$.

$$
\begin{aligned}
& \text { Let } X=\sum_{i \geqq 0} X_{i} \xi^{i+r}, Y=\sum_{j \geqq 0} Y_{j} \xi^{j+r} \text {. Then } \\
& \qquad X Y=\sum X_{i}\left(\begin{array}{c}
i+r \\
s
\end{array}\right) Y_{j}^{(s)} \xi^{i+r-s} \xi^{j+r}=\sum X_{i}\left(\begin{array}{c}
i+r \\
s
\end{array}\right) Y_{j}^{(s)} \xi^{i+j+r-s} \xi^{r},
\end{aligned}
$$

so that

$$
[X, Y]=\left\{\left[\sum\left(\begin{array}{c}
i+r \\
s
\end{array}\right) X_{i} Y_{j}^{(s)}-\sum Y_{j}\left(\begin{array}{c}
j+r \\
s
\end{array}\right) X_{i}^{(s)}\right] \xi^{i+j+r-s}\right\} \xi^{r},
$$

and if $A_{j}$ is the coordinate on $\left(\mathfrak{G}_{\geqq r}\right)^{*}$ dual to $\xi^{j+r}$, we obtain

$$
\begin{gathered}
\sum_{m} A_{m}[X, Y]_{m}=\sum A_{i+j+r-s}\left[\left(\begin{array}{c}
i+r \\
s
\end{array}\right) X_{i} Y_{j}^{(s)}-X_{i}^{(s)}\left(\begin{array}{c}
j+r \\
s
\end{array}\right) Y_{j}\right] \\
\sim \sum_{i, j} X_{i}\left[\sum_{s} A_{i+j+r-s}\left(\begin{array}{c}
i+r \\
s
\end{array}\right) \partial^{s}-\sum_{s}(-\partial)^{s} A_{i+j+r-s}\left(\begin{array}{c}
j+r \\
s
\end{array}\right)\right] Y_{j} .
\end{gathered}
$$

Therefore

$$
B_{i j}^{r}=\sum_{s}\left(\begin{array}{c}
i+r \\
s
\end{array}\right) A_{i+j+r-s} \partial^{s}-\sum_{s}(-\partial)^{s} A_{i+j+r-s}\left(\begin{array}{c}
j+r \\
s
\end{array}\right)
$$


1) Now let $k=1, L$ being given by (2.14), $L^{m}=\sum_{s} p_{s}(m) \xi^{s}$, with $p_{s}$ given by (2.8). We have firstly

$$
\begin{aligned}
L_{\geqq-1, t} & =\left[L, P_{\leqq 0}\right]_{\geqq-1}=\left[L_{>0}, P_{\leqq 0}\right]_{\geqq-1}=\left[\xi^{1} u_{1}, p_{0}(m)+p_{-1}(m) \xi^{-1}+\ldots\right]_{\geqq-1} \\
& =u_{1} \partial p_{0}(m)+\partial\left(u_{1} p_{-1}(m)\right) \xi^{-1}
\end{aligned}
$$

thus

$$
u_{1, t}=0, u_{0, t}=u_{1} \partial p_{0}(m), u_{-1, t}=\partial u_{1} p_{-1}(m)
$$

and now we can as well put $u_{1}=1$. Using (2.8), we obtain

$$
\left(\begin{array}{c}
u_{0} \\
u_{-1}
\end{array}\right)_{t}=\left(\begin{array}{ll}
0 & \partial \\
\partial & 0
\end{array}\right)\left(\begin{array}{c}
\delta / \delta u_{0} \\
\delta / \delta u_{-1}
\end{array}\right)\left(H_{m+1}\right)
$$

Now set $v_{s}=u_{-s-2}, s \geqq 0, P_{\geqq 1}=X=\sum_{s \geqq 0} X_{s} \xi^{s+1}, X_{s}=\frac{\delta H_{m+1}}{\delta v_{s}} ; Y=\sum_{i \geqq 0} Y_{i} \xi^{i+1}$. Then

$$
\begin{aligned}
v_{i, t} & =\operatorname{Res}\left(\xi^{i+1}[X, L]\right)=\operatorname{Res}\left(\xi^{i+1}[X, v]\right)=\sum_{j} B_{i j}\left(X_{j}\right), \\
\bar{Y}^{t} B \bar{X} & =\sum Y_{i} B_{i j}\left(X_{j}\right)=\operatorname{Res}(Y[X, v]) \sim \operatorname{Res}([Y, X] v)=\sum_{j}[Y, X]_{j} v_{j},
\end{aligned}
$$

where $\sum_{j}[Y, X]_{j} \xi^{j+1}=[Y, X]$. Thus $B=B^{1}$, and the (first) Hamiltonian form for the Eq. (2.13) with $k=1$ is

$$
\left(\begin{array}{ll|l}
0 & \partial & \mathbf{0} \\
\partial & 0 & \\
\hline \mathbf{0} & B^{1}
\end{array}\right)
$$

2) Now let $k=2$. Picking out from the equation $L_{t}=\left[L, p_{<2}\right]$ the $\xi^{i}$-terms for $i \geqq-2$, we obtain

$$
\begin{gathered}
u_{1, t}=\left(u_{1} \partial-u_{1}^{(1)}\right) p_{1} ; u_{0, t}=u_{1} \partial p_{0}-\left(u_{0}^{(1)}+u_{1} \partial^{2}\right) p_{1} ; u_{-1, t}=\partial\left(u_{1} p_{-1}-u_{-1} p_{1}\right) \\
u_{-2, t}=\left(\partial u_{1}+u_{1}^{(1)}\right) p_{-2}+\left(u_{0}^{(1)}+\partial^{2} u_{1}\right) p_{-1}-u_{1} \partial p_{0}-\left(u_{2} \partial+\partial u_{2}\right) p_{1},
\end{gathered}
$$

where $p_{i}=p_{i}(m)$ and $u_{i}^{(j)}=\partial^{j}\left(u_{i}\right)$. Using (2.8) we put (2.21) into the form

$$
\begin{aligned}
& \left(\begin{array}{c}
u_{1} \\
u_{0} \\
u_{-1} \\
u_{-2}
\end{array}\right)_{t}=\left(\begin{array}{c|c|c|c}
0 & 0 & 0 & u_{1} \partial-u_{1}^{(1)} \\
\hline 0 & 0 & u_{1} \partial & -u_{1} \partial^{2}-u_{0}^{(1)} \\
\hline 0 & \partial u_{1} & 0 & -\partial u_{-1} \\
\hline \partial u_{1}+u_{1}^{(1)} & \partial^{2} u_{1}+u_{0}^{(1)} & -u_{-1} \partial & -\left(u_{-2} \partial+\partial u_{-2}\right)
\end{array}\right)\left(\begin{array}{c}
\delta / \delta u_{1} \\
\delta / \delta u_{0} \\
\delta / \delta u_{-1} \\
\delta / \delta u_{-2}
\end{array}\right) \\
& \left(H_{m+1}\right) \text {. }
\end{aligned}
$$

Denote $b_{2}$ the matrix in the right-hand side of (2.22). This matrix is Hamiltonian iff the corresponding algebra, say $\mathfrak{h}_{2}$, is a Lie algebra. Computing the commutator in $\mathfrak{h}_{2}$ we find

$$
\begin{aligned}
& \left.\qquad\left[\begin{array}{c}
X_{-2} \\
X_{-1} \\
X_{0} \\
X_{1}
\end{array}\right),\left(\begin{array}{c}
Y_{-2} \\
Y_{-1} \\
Y_{0} \\
Y_{1}
\end{array}\right)\right]=\left(\begin{array}{c}
X_{-2} Y_{-2}^{(1)}-Y_{-2} X_{-2}^{(1)} \\
X_{-2} Y_{-1}^{(1)}-Y_{-2} X_{-1}^{(1)} \\
\partial\left(X_{-2} Y_{0}-Y_{-2} X_{0}\right) \\
A
\end{array}\right), \\
& A:=\left(X_{-2} Y_{1}^{(1)}+2 X_{-2}^{(1)} Y_{1}\right)-\left(Y_{-2} X_{1}^{(1)}+2 Y_{-2}^{(1)} X_{1}\right)+\left(X_{-1}^{(1)} Y_{0}-Y_{-1}^{(1)} X_{0}\right) \\
& +\left(Y_{-2}^{(2)} X_{0}-X_{-2}^{(2)} Y_{0}\right)
\end{aligned}
$$


A straightforward computation shows that the Jacobi identity is indeed satisfied in $\mathfrak{h}_{2}$. (See also Remark 2.27 below.) Thus, $b_{2}$ is Hamiltonian indeed. We now set $v_{s}=u_{-3-s}, s \geqq 0$,

$$
v=\sum_{s \geqq 0} \xi^{-s-3} v_{s}, X=P_{\geqq 2}=\sum_{s \geqq 2} p_{s}(m) \xi^{s}=\sum_{s \geqq 0} X_{s} \xi^{s+2},
$$

where $X_{s}=\delta H_{m+1} / \delta v_{s} ; Y=\sum_{s \geqq 0} Y_{s} \xi^{s+2}$. Then

$$
\begin{gathered}
v_{i, t}=\operatorname{Res}\left(\xi^{i+2}[X, L]\right)=\operatorname{Res}\left(\xi^{i+2}[X, v]\right)=\sum_{j \geqq 0} B_{i j}\left(X_{j}\right), \\
\sum Y_{i} B_{i j}\left(X_{j}\right)=\operatorname{Res}(Y[X, v]) \sim \operatorname{Res}([Y, X] v)=\sum_{j}[Y, X]_{j} v_{j},
\end{gathered}
$$

where $\sum_{j}[Y, X]_{j} \xi^{j+2}=[Y, X]$. Thus, $B=B^{2}$. Therefore, Eqs. (2.13) are Hamiltonian for $k=2$, with the Hamiltonian structure

$$
\left(\begin{array}{c|c}
b_{2} & 0 \\
\hline 0 & B^{2}
\end{array}\right)
$$

This concludes the proof of Theorems 2.5 and 2.1.

Remark 2.25. For the standard case $k=0$, a purely algebraic proof exists (bypassing the Hamiltonian formalism) of the commutativity of all the flows (2.3) [4], which uses the dressing operator $K: K L K^{-1}=u_{n} \xi^{n}$. A somewhat analogous proof can be given for the case $k=1$, but not (I believe) for $k=2$.

Remark 2.26. An analog of the case $k=1$ for discrete integrable systems $[6,9]$ exists with $L=\sum_{-\infty}^{n} u_{i} \zeta^{i}$, where $\zeta$ is an automorphism.

Remark 2.27. For general $n \geqq 1$, the (first) Hamiltonian structure of the system (2.3) can easily be shown to be the direct sum of $B^{k}$ (for the variables $v_{s}=u_{-s-k-1}, s \geqq 0$ ) and the natural Hamiltonian matrix on the dual space to the Lie factor-algebra $\left(\mathfrak{b}_{<k} / \mathfrak{b}_{<-n-1}\right.$ (for the variables $u_{-k}, \ldots, u_{-n}$ ) specialized by the normalization conditions (2.2).

Remark 2.28. Substituting the Poisson bracket $\{a, b\}=a_{\xi} b_{x}-a_{x} b_{\xi}$ instead of the commutator into the right-hand side of (2.3), we arrive at the dispersiveless integrable systems which can be considered as the quasiclassical limits of the full Eq. (2.3). The resulting flows all commute, have an infinity of common c.l's, and are Hamiltonian with the Hamiltonian structures which are the limits of the corresponding structures for the full equations.

Remark 2.29. It would be interesting to find out whether the second Hamiltonian form exists for the Eq. (2.3) when $k \neq 0$. For $k=0$ it is not very difficult to show that it does exist (the quasi-classical limit of this structure, written down in [10], represents the second Hamiltonian form of the two-dimensional, free surface, long wave Eqs. [11-13]).

Remark 2.30. Any time a new integrable system, or class of systems, is introduced one has to tackle the (often overlooked) problem of triviality or nontriviality of flows and conservation laws. For complex systems the only known general avenue 
to analyze this problem is first solving the easier problem for the flows and then using the Hamiltonian formalism to analyze the conservation laws (see, e.g., $[17,18])$. For the system (2.3) the answer is obvious: $\operatorname{Res} L^{m / n} \nsim 0$ for $m>0$ unless $k=0$ and $m \in \mathbb{N} n$, since the quasiclassical limit of $\operatorname{Res} L^{m / n}$ is simply a polynomial in $u_{i}$ 's with positive binomial coefficients, except for $k=0$ and $\left(L^{m / n}\right)_{<0}=0$, that is, when $m \in \mathbb{N} n$. The nontriviality of flows follows by similar arguments: all the flows are nontrivial except for $k=0$ and $P=L^{m / n}$ with $m \in \mathbb{N} n$.

\section{Hamiltonian Formalism for Dispersive Long Waves}

In this section we prove Theorem 3.0. Retaining the notation $L=\xi+u+h \xi^{-1}$, $L^{m}=\sum_{s} \xi^{s} p_{s}(m), H_{m}=m^{-1} \operatorname{Res} L^{m}$, we have by (2.8)

$$
p_{0}(m)=\delta H_{m+1} / \delta h, p_{-1}(m)=\delta H_{m+1} / \delta u .
$$

Picking out the $\xi^{0}$ - and $\xi^{1}$-terms in the equality $L_{t}=\left[L,\left(\left(P^{\dagger}\right)_{\leqq 0}\right)^{\dagger}\right]$, we have

$$
L_{t}=u_{t}+h_{t} \xi^{-1}=\left[\xi+u+h \xi^{-1}, \sum_{s \leqq 0} \xi^{s} p_{s}(m)\right]_{\geqq-1}=\partial p_{0}(m)+\partial p_{-1}(m) \xi^{-1},
$$

so that

$$
\left(\begin{array}{l}
u \\
h
\end{array}\right)_{t}=\partial\left(\begin{array}{c}
p_{0}(m) \\
p_{-1}(m)
\end{array}\right)=\left(\begin{array}{ll}
0 & \partial \\
\partial & 0
\end{array}\right)\left(\begin{array}{l}
\delta / \delta u \\
\delta / \delta h
\end{array}\right)\left(H_{m+1}\right),
$$

which yields (1.9).

We now write down the identity $\left(L^{\dagger}\right)^{m+1}=\left(L^{\dagger}\right)^{m} L^{\dagger}=L^{\dagger}\left(L^{\dagger}\right)^{m}$ :

$$
\begin{aligned}
\sum_{s}(-1)^{s} p_{s}(m+1) \xi^{s} & =\sum_{s}(-1)^{s} p_{s}(m) \xi^{s}\left(-\xi+u-\xi^{-1} h\right) \\
& =\left(-\xi+u-\xi^{-1} h\right) \sum_{s}(-1)^{s} p_{s}(m) \xi^{s},
\end{aligned}
$$

and pick out the $\xi^{i}$-terms from it, for $i=0,-1,-2$ :

$$
\begin{gathered}
p_{0}(m+1)=p_{-1}(m)-\partial p_{0}(m)+u p_{0}(m)+\sum_{s \geqq 0}\left[h p_{s+1}(m)\right]^{(s)}, \\
-p_{-1}(m+1)=-p_{-2}(m)-p_{-1}(m) u-p_{0}(m) h, \\
-p_{-1}(m+1)=-p_{-2}(m)+\partial p_{-1}(m)-u p_{-1}(m)-\sum_{s \geqq 0}\left[h p_{s}(m)\right]^{(s)}, \\
p_{-2}(m+1)=p_{-3}(m)+p_{-2}(m) u+p_{-1}(m) \partial u+p_{-1}(m) h+p_{0}(m) \partial h, \\
p_{-2}(m+1)=p_{-3}(m)-\partial p_{-2}(m)+u p_{-2}(m)+\sum_{s \geqq-1}\left[h p_{s}(m)\right]^{(s+1)} .
\end{gathered}
$$

Comparing (3.5) with (3.6) we obtain

$$
p_{-1}(m)=\sum_{s \geqq 0}\left[h p_{s+1}(m)\right]^{(s)},
$$

and substituting this into (3.4) we get

$$
p_{0}(m+1)=2 p_{-1}(m)+(u-\partial) p_{0}(m) .
$$

In particular,

$$
\partial p_{0}(m+1)=2 \partial p_{-1}(m)+\partial(u-\partial) p_{0}(m)
$$


Subtracting (3.8) from (3.7) we obtain

$$
\begin{aligned}
\partial p_{-2}(m) & +p_{-1}(m) \partial u+p_{0}(m) \partial h=\sum_{s \geqq 0}\left[h p_{s}(m)\right]^{(s+1)}=\partial h p_{0}(m) \\
& +\partial^{2} \sum_{s \geqq 0}\left[h p_{s+1}(m)\right]^{(s)}=[\text { by }(3.9)]=\partial h p_{0}(m)+\partial^{2} p_{-1}(m) .
\end{aligned}
$$

Applying ( $-\partial$ ) to (3.5) and substituting (3.12) into the result, we arrive at

$$
\partial p_{-1}(m+1)=\left(u \partial+\partial^{2}\right) p_{-1}(m)+(h \partial+\partial h) p_{0}(m) \text {. }
$$

Combining (3.11) and (3.13) together, we get

$$
\left(\begin{array}{l}
u \\
h
\end{array}\right)_{t}=\partial\left(\begin{array}{c}
p_{0}(m) \\
p_{-1}(m)
\end{array}\right)=\left(\begin{array}{cc}
2 \partial & \partial(u-\partial) \\
(u+\partial) \partial & h \partial+\partial h
\end{array}\right)\left(\begin{array}{c}
p_{-1}(m-1) \\
p_{0}(m-1)
\end{array}\right),
$$

which proves $(1.10)$. Notice that only $\partial p_{-1}(m-1)$ is involved in the right-hand side of (3.14). We, thus, can iterate the procedure using (3.10) and (3.11):

$$
\begin{aligned}
u_{t}=2 \partial p_{-1}(m+1)+\partial(u-\partial) p_{0}(m-1) \\
=2\left[\left(u \partial+\partial^{2}\right) p_{-1}(m-2)+(h \partial+\partial h) p_{0}(m-2)\right] \\
+\partial(u-\partial)\left[2 p_{-1}(m-2)+(u-\partial) p_{0}(m-2)\right] \\
=2(u \partial+\partial u) p_{-1}(m-2)+\left[2(h \partial+\partial h)+\partial(u-\partial)^{2}\right] p_{0}(m-2), \\
h_{t}=\left(u \partial+\partial^{2}\right) p_{-1}(m-1)+(h \partial+\partial h) p_{0}(m-1) \\
=(u+\partial)\left[(u+\partial) \partial p_{-1}(m-2)+(h \partial+\partial h) p_{0}(m-2)\right] \\
+(h \partial+\partial h)\left[2 p_{-1}(m-2)+(u-\partial) p_{0}(m-2)\right] \\
=\left[(u+\partial)^{2} \partial+2(h \partial+\partial h)\right] p_{-1}(m-2) \\
+ \\
+(u+\partial)(h \partial+\partial h)+(h \partial+\partial h)(u-\partial)] p_{0}(m-2),
\end{aligned}
$$

which proves (1.11) for $m>1$ : for $m=1, p_{-1}(1)$ and $p_{0}(-1)$ are not the variational derivatives of $H_{0}$ which is not defined. To fix this, we define $H_{0}=u / 2$. Then (3.1) implies

$$
p_{-1}(-1)=1 / 2, p_{0}(-1)=0 \text {. }
$$

Substituting this into (3.15) we obtain

$$
u_{t}=\partial u, h_{t}=\partial h
$$

which agrees with (3.2) for $m=1$ since $p_{0}(1)=u$ and $p_{-1}(1)=h$.

We now prove that the matrices (1.9)-(1.11) are Hamiltonian.

The matrix $B^{\mathrm{I}}(1.9)$ is skew-symmetric constant-coefficient and is, thus, Hamiltonian [5].

Let $K$ be a differential algebra with a derivation $\partial$; denote $D(K) K$ considered as a Lie algebra with the commutator $[X, Y]=X \partial Y-Y \partial X . D(K)$ acts on $K$ by derivations: $(X, f) \mapsto X \partial f$. Denote the corresponding semidirect product Lie algebra by $\mathfrak{h}: \mathfrak{h}=D(K) \odot K$. The commutator in $\mathfrak{h}$ is given by

$$
\left[\left(\begin{array}{l}
X \\
f
\end{array}\right),\left(\begin{array}{l}
Y \\
g
\end{array}\right)\right]=\left(\begin{array}{c}
X \partial Y-Y \partial X \\
X \partial g-Y \partial f
\end{array}\right) \text {. }
$$


Let $h$ and $u$ denote coordinates on $\mathfrak{h}^{*}$ dual to $D(K)$ and $K$ respectively. Then

$$
h(X \partial Y-Y \partial X)+u(X \partial g-Y \partial f) \sim(f, X)\left(\begin{array}{cc}
0 & \partial u \\
u \partial & h \partial+\partial h
\end{array}\right)\left(\begin{array}{l}
g \\
Y
\end{array}\right),
$$

hence the linear part $B^{\mathrm{IIl}}$ of $B^{\mathrm{II}}$ is the natural Hamiltonian matrix on $\mathfrak{h}^{*}[6-8]$. Consider now the following skew-symmetric forms on $\mathfrak{h}$ :

$$
\begin{gathered}
\omega_{1}\left(\left(\begin{array}{l}
f \\
X
\end{array}\right),\left(\begin{array}{l}
g \\
Y
\end{array}\right)\right)=2 f \partial g=(f, X) b_{1}(g, Y)^{t}, b_{1}=\left(\begin{array}{cc}
2 \partial & 0 \\
0 & 0
\end{array}\right) \\
\omega_{2}\left(\left(\begin{array}{c}
f \\
X
\end{array}\right),\left(\begin{array}{l}
g \\
Y
\end{array}\right)\right)=X \partial^{2} g-f \partial^{2} Y=(f, X) b_{2}(g, Y)^{t}, b_{2}=\left(\begin{array}{cc}
0 & -\partial^{2} \\
\partial^{2} & 0
\end{array}\right) .
\end{gathered}
$$

It is easy to see that $\omega_{1}$ and $\omega_{2}$ are generalized two-cocycles on hy, i.e., $\omega_{i}\left(\left[A_{1}, A_{2}\right]\right.$, $\left.A_{3}\right)+$ c.p. $\sim 0, \forall A_{1}, A_{2}, A_{3} \in \mathfrak{h}, i=1,2$, where "c.p." stands for "cyclic permutation." Thus $[6,7]$, the matrix $B^{\mathrm{II}}=B^{\mathrm{IIl}}+b_{1}+b_{2}$ is Hamiltonian.

The Hamiltonian property of the matrix $B^{\mathrm{III}}$ can be checked directly, by a tedious but straightforward computation, using methods of [13] (see, e.g., [14]). Alternatively, consider the following matrices over $C_{2}=C_{U, v}=\mathbb{C}\left[U^{(i)}, v^{(j)}\right]$ :

$$
\begin{gathered}
\bar{B}^{\mathbb{I}}=\left(\begin{array}{cc}
2 \partial & \partial \\
\partial & 0
\end{array}\right), \\
\bar{B}^{\mathrm{II}}=\left(\begin{array}{cc}
2(U \partial+\partial U) & 2 v \partial+\partial U-\partial^{2} \\
2 \partial v+U \partial+\partial^{2} & v \partial+\partial v
\end{array}\right) .
\end{gathered}
$$

Let $\Phi: C_{1}=C_{u, h} \rightarrow C_{2}$ be the differential homomorphism of differential algebras given on generators $u, h$ by the formula

$$
\Phi(u)=U, \Phi(h)=U v-v^{2}+v^{(1)} .
$$

Let $J=J(\Phi)$ be the Fréchet Jacobian of $\Phi$ :

$$
J=\left(\begin{array}{cc}
1 & 0 \\
v & U-2 v+\partial
\end{array}\right) .
$$

It is easy to check out that

$$
J \bar{B}^{i} J^{\dagger}=\Phi\left(B^{i+\mathrm{I}}\right), \quad i=\mathrm{I}, \mathrm{II} .
$$

In addition, the map $\Phi$ (3.24) is obviously injective (this also follows from a more general injectivity result from [15]). This implies that the matrix $B^{i+1}$ is Hamiltonian provided $\bar{B}^{i}$ is (since $B^{i+1}$ is just the reduction of $\bar{B}^{i}$ on the image $\Phi\left(C_{1}\right) \subset C_{2}$, see [15]).

The matrix $\bar{B}^{1}$ is skew-symmetric constant-coefficient, and thus is Hamiltonian. It is easy to associate a generalized 2-cocycle and a Lie algebra to the matrix $\bar{B}^{\text {II }}$ (3.23). Instead, let us consider two more differential algebras $C_{3}=C_{\varphi, V}$ and $C_{4}=C_{w, \psi}$, together with the following matrices $b_{3}$ and $b_{4}$ over $C_{3}$ and $C_{4}$ respectively:

$$
\begin{gathered}
b_{4}=\left(\begin{array}{cc}
2(w \partial+\partial w) & -2 \psi^{(1)}+\partial \\
2 \psi^{(1)}+\partial & 0
\end{array}\right), \\
b_{3}=\left(\begin{array}{cc}
0 & -\partial \\
-\partial & 0
\end{array}\right) .
\end{gathered}
$$


Let $\varphi_{3}: C_{2} \rightarrow C_{3}$ and $\varphi_{4}: C_{2} \rightarrow C_{4}$ be the differential homomorphisms given by

$$
\begin{aligned}
& \varphi_{3}(U)=\varphi^{(1)}+2 V, \varphi_{3}(v)=V, \\
& \varphi_{4}(U)=w, \varphi_{4}(v)=\psi^{(1)}+\frac{1}{2} w .
\end{aligned}
$$

The maps $\varphi_{3}, \varphi_{4}$ are evidently injective. If $J_{j}$ is the Fréchet Jacobian of the map $\varphi_{j}$, $j=3,4$, one can easily check out that

$$
J_{j} b_{j} J_{j}^{\dagger}=\varphi_{j}\left(\bar{B}^{\mathrm{II}}\right) .
$$

Since $b_{3}$ is obviously Hamiltonian, it follows that $\bar{B}^{\mathrm{II}}$ is Hamiltonian too, and, thus, $B^{\mathrm{III}}$ is Hamiltonian as well. Theorem 3.0 is proved.

The matrices $\bar{B}^{\mathrm{I}}, \bar{B}^{\mathrm{II}}, b_{3}$, and $b_{4}$ have the following interpretation. $U$ and $v$ are the variables of the modified long wave hierarchy

$$
\left(\begin{array}{l}
U \\
v
\end{array}\right)_{t}=\bar{B} \delta\left(\Phi H_{m}\right)=\bar{B}^{\mathrm{II}} \delta\left(\Phi H_{m-1}\right)
$$

which is, thus, integrable and bi-Hamiltonian. The canonical map $\Phi$ is an analog of the Miura map. In addition, we have two different Hamiltonian modified-modified systems

$$
\left(\begin{array}{l}
\varphi \\
V
\end{array}\right)_{t}=b_{3} \delta\left(\varphi_{3} \Phi H_{m-1}\right),\left(\begin{array}{l}
w \\
\psi
\end{array}\right)_{t}=b_{4} \delta\left(\varphi_{4} \Phi H_{m-1}\right) .
$$

This phenomenon is absent in the standard theory of integrable systems $(k=0)$; neither three-Hamiltonian systems are present when $k=0$.

It remains to show that $b_{4}$ is Hamiltonian. Let $\mathfrak{h}_{1}$ be the semidirect product Lie algebra $D(K) \odot K$, where $D(K)$ acts now on $K$ via the rule $(X, f) \mapsto \partial(X f)$. Thus, the commutator in $\mathfrak{h}_{1}$ is

$$
\left[\left(\begin{array}{l}
X \\
f
\end{array}\right),\left(\begin{array}{l}
Y \\
g
\end{array}\right)\right]=\left(\begin{array}{c}
X \partial Y-Y \partial X \\
\partial(X g-Y f)
\end{array}\right) .
$$

Let $w$ and $\psi$ be the corresponding coordinates on $\mathfrak{h}_{1}^{*}$. Then

$$
w(X \partial Y-Y \partial X)+\psi \partial(X g-Y f) \sim(X, f)\left(\begin{array}{cc}
w \partial+\partial w & -\psi_{x} \\
\psi_{x} & 0
\end{array}\right)\left(\begin{array}{l}
Y \\
g
\end{array}\right),
$$

and hence the linear part $b_{4}^{l}$ of $b_{4}$ is twice the natural Hamiltonian matrix on $\mathfrak{h}_{1}^{*}$. Consider the following skew-symmetric form on $\mathfrak{h}_{1}$ :

$$
\omega\left(\left(\begin{array}{l}
X \\
f
\end{array}\right),\left(\begin{array}{l}
Y \\
g
\end{array}\right)\right)=X \partial g+f \partial Y=(X, f)\left(\begin{array}{ll}
0 & \partial \\
\partial & 0
\end{array}\right)\left(\begin{array}{l}
Y \\
g
\end{array}\right) .
$$

This form is clearly seen to be a generalized two-cocycle on $\mathfrak{h}_{1}$. Hence $b_{4}$ is indeed Hamiltonian.

Remark 3.37. Not only matrices $B^{\mathrm{I}}, B^{\mathrm{II}}$, and $B^{\mathrm{III}}$ are individually Hamiltonian, but an arbitrary linear combination $\sum_{\mathrm{I}}^{\mathrm{III}} \lambda_{i} B^{i}$ is Hamiltonian as well. This follows by the 
same line of reasoning as above, when combined with the obvious formula

$$
B^{\mathrm{III}}(u+\lambda)=B^{\mathrm{III}}(u)+2 \lambda B^{\mathrm{II}}(u)+\lambda^{2} B^{\mathrm{I}}, \lambda_{i}, \lambda \in \mathbb{C} .
$$

Remark 3.38. For arbitrary $\alpha$ and $\beta$ in (1.2), all the results in this section apply after a slight change is made in matrices $B^{i}, \bar{B}^{i}, b_{3}, b_{4}$ and the maps $\Phi, \varphi_{3}, \varphi_{4}$. For example, for $\beta=-1 / 2$, the corresponding matrix $B^{\mathrm{III}}(1.11)$ adds on $2 \alpha\left(\begin{array}{cc}0 & 2 \partial^{3} \\ 2 \partial^{3} & u \partial^{3}+\partial^{3} u\end{array}\right)$.

Remark 3.39. Under zero-dispersion limit the dispersive system (1.7) goes into the classical long wave system (1.1), while the limit of the matrices $B^{\mathrm{I}}, B^{\mathrm{II}}$, and $B^{\mathrm{III}}$ produces the corresponding three-Hamiltonian form of this system ([16]).

\section{Canonical Maps}

Suppose $B_{1}$ and $B_{2}$ are Hamiltonian matrices over differential rings $C_{1}$ and $C_{2}$ respectively and let $\Phi: C_{1} \rightarrow C_{2}$ be a differential homomorphism. $\Phi$ is called canonical if $\Phi X_{H}=X_{\Phi H} \Phi$, for any $H \in C_{1}$; here $X_{G}$ is the Hamiltonian evolution derivation whose Hamiltonian is $G$. If $J=J(\Phi)$ is the Fréchet Jacobian of $\Phi$, then the condition on $\Phi$ to be canonical can conveniently be written in the form (see $[6,15])$

$$
J B_{2} J^{\dagger}=\Phi\left(B_{1}\right) .
$$

We fix $r \in \mathbb{Z}_{+}$. Let $C_{2}=C_{u, h}$, and define $B_{2}$ as

$$
B_{2}=B_{2}(r)=\left(\begin{array}{cc}
0 & \partial(\partial+u)^{r} \\
(u-\partial)^{r} \partial & h(\partial+u)^{r}-(u-\partial)^{r} h
\end{array}\right) .
$$

Let $B_{1}=B^{r}$ be the natural Hamiltonian matrix $(2.17)$ on the dual space $\left(\mathfrak{G}_{\geq r}\right)^{*}$ to the Lie algebra of differential operators of order $\geqq r$. For each $i \in \mathbb{Z}_{+}$define $Q_{i}=Q_{i}(u) \in C_{u}$ by

$$
Q_{i}=(\partial+u)^{i}(1) .
$$

Theorem 4.4. The map $\Phi: C_{1}=C_{A}=\mathbb{C}\left[A_{i}^{(j)}\right] \rightarrow C_{2}=C_{u, h}$ given by

$$
\Phi\left(A_{i}\right)=h Q_{i}(u)
$$

is canonical between $B_{2}(r)$ (4.2) and $B^{r}$ (2.17).

Remark 4.6. Let $\varphi: C_{2} \rightarrow C_{1}$ be the following rational map: $\varphi(h)=A_{0}$, $\varphi(u)=A_{1} / A_{0}$. Then $\Phi \varphi=i d$. Thus $\varphi$ is an epimorphism. Since $B^{r}$ is Hamiltonian, from Theorem 4.4 it would follow that $B_{2}(r)$ is Hamiltonian too.

Before proving Theorem 4.4, we record a few properties of the polynomials $Q_{i}$ (4.3). Notice, that by (4.3),

$$
Q_{i+1}=(\partial+u)\left(Q_{i}\right), Q_{0}=1 .
$$

Lemma 4.8. For $m \in \mathbb{Z}$,

$$
(\xi+u)^{m}=\sum_{\alpha \geqq 0}\left(\begin{array}{c}
m \\
\alpha
\end{array}\right) Q_{\alpha} \xi^{m-\alpha} .
$$


Proof. We use induction on $|m|$. Let first $m \geqq 0$. For $m=0(4.9)$ is obviously true. If it is true for $0 \leqq m \leqq M$, then

$$
\begin{aligned}
(\xi+u)^{M+1} & =(\xi+u)(\xi+u)^{M}=(\xi+u) \sum\left(\begin{array}{c}
M \\
\alpha
\end{array}\right) Q_{\alpha} \xi^{M-\alpha} \\
& =\sum\left(\begin{array}{c}
M \\
\alpha
\end{array}\right)\left[(\partial+u)\left(Q_{\alpha}\right)+Q_{\alpha} \xi\right] \xi^{M-\alpha} \\
& =\sum\left(\begin{array}{c}
M \\
\alpha
\end{array}\right) Q_{\alpha+1} \xi^{M-\alpha}+\sum\left(\begin{array}{c}
M \\
\alpha
\end{array}\right) Q_{\alpha} \xi^{M+1-\alpha} \\
& =\Sigma\left[\left(\begin{array}{c}
M \\
\alpha-1
\end{array}\right)+\left(\begin{array}{c}
M \\
\alpha
\end{array}\right)\right] Q_{\alpha} \xi^{M+1-\alpha}=\sum\left(\begin{array}{c}
M+1 \\
\alpha
\end{array}\right) Q_{\alpha} \xi^{M+1-\alpha},
\end{aligned}
$$

and the induction step is made.

$$
\begin{aligned}
\text { Now let } m=-1 \text {. Since }\left(\begin{array}{c}
-1 \\
\alpha
\end{array}\right) & =(-1)^{\alpha},(4.9) \text { becomes } \\
(\xi+u)^{-1} & =\sum_{\alpha \geqq 0}(-1)^{\alpha} Q_{\alpha} \xi^{-\alpha-1} .
\end{aligned}
$$

To check (4.10) we apply $\xi+u$ to the right-hand side of (4.10):

$$
(\xi+u) \sum(-1)^{\alpha} Q_{\alpha} \xi^{-\alpha-1}=\sum(-1)^{\alpha}\left[Q_{\alpha+1} \xi^{-\alpha-1}+Q_{\alpha} \xi^{-\alpha}\right]=Q_{0} \xi^{-0}=1,
$$

which proves (4.10). Now suppose (4.9) is true for $0>m \geqq-M$, and let

$$
(\xi+u)^{-M-1}=\sum_{\alpha \geqq 0}\left(\begin{array}{c}
-M-1 \\
\alpha
\end{array}\right) F_{\alpha} \xi^{-M-1-\alpha}
$$

with some $F_{\alpha}$. To prove that $F_{\alpha}=Q_{\alpha}$, we apply $\xi+u$ to (4.11) and get

$$
(\xi+u)^{-M}=\sum_{\alpha \geqq 0}\left(\begin{array}{c}
-M-1 \\
\alpha
\end{array}\right)\left[(\partial+u)\left(F_{\alpha}\right) \xi^{-M-1-\alpha}+F_{\alpha} \xi^{-M-\alpha}\right] .
$$

But

$$
(\xi+u)^{-M}=\sum_{\alpha \geqq 0}\left(\begin{array}{c}
-M \\
\alpha
\end{array}\right) Q_{\alpha} \xi^{-M-\alpha}
$$

by induction assumption. Equating $\xi^{-M-\alpha}$-terms in (4.12) and (4.13) we obtain $F_{0}=Q_{0}$ and

$$
\left(\begin{array}{c}
-M-1 \\
\alpha+1
\end{array}\right) F_{\alpha+1}=\left(\begin{array}{c}
-M \\
1+\alpha
\end{array}\right) Q_{\alpha+1}-\left(\begin{array}{c}
-M-1 \\
\alpha
\end{array}\right)(\partial+u)\left(F_{\alpha}\right) .
$$

Since

$$
\left(\begin{array}{c}
-M-1 \\
1+\alpha
\end{array}\right)+\left(\begin{array}{c}
-M-1 \\
\alpha
\end{array}\right)=\left(\begin{array}{c}
-M \\
\alpha+1
\end{array}\right)
$$

the induction on $\alpha$ for (4.14) shows that $F_{\alpha}=Q_{\alpha}$.

\section{Corollary 4.15.}

$$
(\xi-u)^{-1}=\sum_{i \geqq 0} \xi^{-i-1} Q_{i}
$$

Proof. Take adjoint of (4.10). 
Lemma 4.17. For $m, r \in \mathbb{Z}_{+}$,

$$
\sum_{\alpha \geqq 0}\left(\begin{array}{c}
m \\
\alpha
\end{array}\right) Q_{m+r-\alpha} \xi^{\alpha}=\sum_{\alpha \geqq 0}\left(\begin{array}{c}
m \\
\alpha
\end{array}\right) Q_{m-\alpha} \xi^{\alpha} Q_{r}
$$

Proof. We use induction on $m$. For $m=0,(4.18)$ is obviously true. Assume it is true for $0 \leqq m \leqq M$. Noticing that the right-hand side of (4.18) can be written as $(\xi+u)^{m} Q_{r}$ by (4.9), we have

$$
\begin{aligned}
(\xi+u)^{M+1} Q_{r} & =(\xi+u)(\xi+u)^{M} Q_{r}=(\xi+u)^{M}(\xi+u) Q_{r} \\
& =(\xi+u)^{M}\left(Q_{r+1}+Q_{r} \xi\right)=[\text { by }(4.18) \text { with } m=M] \\
& =\sum\left(\begin{array}{c}
M \\
\alpha
\end{array}\right) Q_{M+r+1-\alpha} \xi^{\alpha}+\sum\left(\begin{array}{c}
M \\
\alpha
\end{array}\right) Q_{M+r-\alpha} \xi^{\alpha+1} \\
& =\sum\left[\left(\begin{array}{c}
M \\
\alpha
\end{array}\right)+\left(\begin{array}{c}
M \\
\alpha-1
\end{array}\right)\right] Q_{M+r+1-\alpha} \xi^{\alpha}=\sum\left(\begin{array}{c}
M+1 \\
\alpha
\end{array}\right) Q_{M+1+r-\alpha} \xi^{\alpha},
\end{aligned}
$$

which finishes the induction step.

Denote $D\left(Q_{m}\right)=D_{u}\left(Q_{m}\right)=\sum_{i \geqq 0} \frac{\partial Q_{m}}{\partial u^{(i)}} \xi^{i}$ the Fréchet derivative of $Q_{m}$ with respect
to $u$.

\section{Lemma 4.19.}

$$
D\left(Q_{m}\right) \xi+Q_{m}=(\xi+u)^{m} .
$$

Proof. Use induction on $m$, (4.20) being true for $m=0$. Suppose (4.20) is true for $0 \leqq m \leqq M$. Recall that the operator $D$ is a derivation: $D(A B)=A D(B)+B D(A)$. Applying $D$ to (4.7), we obtain

$$
D\left(Q_{m+1}\right)=(\xi+u) D\left(Q_{m}\right)+Q_{m}
$$

therefore

$$
\begin{aligned}
D & \left(Q_{M+1}\right) \xi+Q_{M+1}=(\xi+u) D\left(Q_{M}\right) \xi+Q_{M} \xi+Q_{M+1} \\
& =[\text { by induction assumption }] \\
& =(\xi+u)\left[(\xi+u)^{M}-Q_{M}\right]+Q_{M} \xi+u Q_{M}+\partial\left(Q_{M}\right)=(\xi+u)^{M+1} .
\end{aligned}
$$

Proof of Theorem 4.4. From (4.5) we get, denoting $\varphi_{i}=\Phi\left(A_{i}\right)$ :

$$
D_{u}\left(\varphi_{i}\right)=h D\left(Q_{i}\right), D_{h}\left(\varphi_{i}\right)=Q_{i} .
$$

Taking (ij)-entry of the left-hand side of (4.1) we obtain, using (4.2) and (2.17)

$$
\begin{aligned}
h & {\left[D\left(Q_{i}\right) \xi+Q_{i}\right](\xi+u)^{r} Q_{j}-Q_{i}(u-\xi)^{r}\left[Q_{j}-\xi D\left(Q_{j}\right)^{\dagger}\right] h } \\
& =[\text { by }(4.20) \text { and its adjoint }] \\
& =h(\xi+u)^{r+i} Q_{j}-Q_{i}(u-\xi)^{r+j} h=[\text { by }(4.18) \text { and its adjoint }] \\
& =h \sum_{\alpha}\left(\begin{array}{c}
r+i \\
\alpha
\end{array}\right) Q_{i+r+j-\alpha} \xi^{\alpha}-\sum_{\alpha}(-\xi)^{\alpha}\left(\begin{array}{c}
r+j \\
\alpha
\end{array}\right) Q_{r+j+i-\alpha} h \\
& =[\text { by }(2.17),(4.5)]=\Phi\left(B_{i j}^{r}\right) .
\end{aligned}
$$


Proof of Theorem 4.0. The change of $u$ into $-u$ amounts to considering $\left(\xi+u+h \xi^{-1}\right)^{\dagger}$ instead of $\xi+u+h \xi^{-1}$. Thus, put $L=\xi+u+\xi^{-1} h$. Notice that for any pseudo-differential operator $X=\sum a_{i} \xi^{i}, a_{i} \in$ some $C$, and any $v \in C$, $\operatorname{Res} X=\operatorname{Res} \sum a_{i}(\xi-v)^{i}$. Thus,

$$
\begin{aligned}
\operatorname{Res} L^{m} & =\operatorname{Res}\left[\xi+(\xi-u)^{-1} h\right]^{m}=[\text { by }(4.16)] \\
& =\operatorname{Res}\left(\xi+\sum \xi^{-i-1} Q_{i} h\right)^{m}=\Phi \operatorname{Res}\left(\xi+\sum \xi^{-i-1} A_{i}\right)^{m}
\end{aligned}
$$

which proves (ii).

Now notice that the change $u \rightarrow-u$ does not change the matrix $B^{\mathrm{I}}(1.9)$. By Theorem 4.4 with $r=0$, the map $\Phi: \Phi\left(A_{i}\right)=h Q_{i}$ is canonical between the Hamiltonian structure $B^{\mathrm{I}}(1.9)$ of Eqs. (1.4) and the matrix $B^{0}(2.16)$ representing the Hamiltonian structure of Eq. (2.13) with $k=0$. Therefore, the corresponding Hamiltonian derivations in $C_{u, h}$ and $C_{A}$ are compatible for $\Phi$-connected Hamiltonians, and (4.21) shows that the Hamiltonians are compatible indeed. This proves (i).

\section{Specializations}

In this section we prove Theorem 5.0 and place it into a general context of specializations of the system (2.3).

Using the notation of Sect. 3, we rewrite (3.15a) using (3.2):

$$
\partial p_{0}(m)=2(u \partial+\partial u) p_{-1}(m-2)+\left[2(h \partial+\partial h)+\partial(u-\partial)^{2}\right] p_{0}(m-2) .
$$

Corollary 5.2. If $u=0$, that is,

$$
L=\xi+h \xi^{-1}
$$

then

$$
p_{0}(2 m+1)=0, \quad m \in \mathbb{Z}_{+} .
$$

Proof. Since $p_{0}(1)=0$, induction on $m$ with the help of (5.1) yields $\left\{p_{0}(2 m-1)=0 \Rightarrow \partial p_{0}(2 m+1)=0\right\} \quad$ which implies $p_{0}(2 m+1)=0$, since $r k p_{s}(m)=m-s$ with $r k h^{(j)}=j+2, r k \mathbb{C}=0$.

Corollary 5.5. The equation

$$
L_{t}=\left[\left(\left(P^{\dagger}\right)_{\geqq 1}\right)^{\dagger}, L\right], P=L^{2 m+1}, L=\xi+h \xi^{-1},
$$

is meaningful, that is, it preserves the subring $C_{h} \subset C_{u, h}$.

Proof. By (3.2), $u_{t}=\partial p_{0}(2 m+1)$ for the full system (1.4), and we have just seen that $p_{0}(2 m+1)=0$.

For the variable $h$, we use (3.2) and (3.15b):

$$
h_{t}=\partial p_{-1}(2 m+1)=\left[\partial^{3}+2(h \partial+\partial h)\right] p_{-1}(2 m+1) .
$$

On the other hand, $2 p_{-1}(2 m-1)=p_{0}(2 m)$ by $(3.10)$, and $p_{0}(2 m)=\delta H_{2 m+1} / \delta h$ by (3.1), since letting $u$ vanish does not interfere with taking the variational derivative with respect to $h$. Thus,

$$
h_{t}=\frac{1}{2} \partial \frac{\delta H_{2 m+3}}{\delta h}=\left[\frac{1}{2} \partial^{3}+h \partial+\partial h\right] \frac{\delta H_{2 m+1}}{\delta h},
$$


which proves the (ii) part of Theorem 5.0. Notice that $H_{2 m} \sim 0$ by (3.1), since $p_{0}(2 m-1)=0$ by (5.4). On the other hand, $H_{2 m+1} \nsim 0$, since the quasiclassical limit of $H_{2 m+1}$ is $\frac{1}{2 m+1}\left(\begin{array}{c}2 m+1 \\ m\end{array}\right) h^{m+1} \nsim 0$. Now, $H_{3} \sim h^{2}$, so (5.8) yields

$$
h_{t}=h_{x x x}+6 h h_{x} \text {, }
$$

which is the $\mathrm{KdV}$ equation. Comparing (5.7) with (1.25) we see that each of Eqs. (5.6) differs by a constant multiple from the corresponding member of the $\mathrm{KdV}$ hierarchy. This proves the (i) part of Theorem 5.0.

The possibility of the specialization $u=0$ for the operator $\xi+u+h \xi^{-1}$ is a particular instance of the following general picture.

Theorem 5.10. Let $L$ be given as

$$
L=\sum^{n} \xi^{i} u_{i}
$$

Then the equations

$$
L_{t}=\left[P_{\geqq k}, L\right], P=L^{m / n}
$$

preserve the relation

$$
L^{\dagger}=(-1)^{n} \xi^{k} L \xi^{-k}
$$

for

$$
m=2 M+1, \quad M \in \mathbb{Z}_{+}
$$

(recall that $k=0,1$ or 2 ).

Proof. Since (5.13) is equivalent to $\mathscr{L}^{\dagger}=-\xi^{k} \mathscr{L} \xi^{-k}$ for $\mathscr{L}=L^{1 / n}$, we can restrict ourselves to the case $n=1$ only.

\section{Lemma 5.15. Suppose}

$$
\begin{gathered}
L=\sum^{1} \xi^{i} u_{i} ; P=L^{m}=\sum_{s} p_{s}(m) \xi^{s}, \quad m \in \mathbb{Z}_{+} ; \\
L^{\dagger}=-\xi^{r} L \xi^{-r}, \quad r \in \mathbb{Z} .
\end{gathered}
$$

Then

$$
\begin{gathered}
\left(P \xi^{-r}\right)^{\dagger}=(-1)^{m+r} P \xi^{-r}, \\
\left(P_{\geqq r} \xi^{-r}\right)^{\dagger}=(-1)^{m+r} P_{\geqq r} \xi^{-r} .
\end{gathered}
$$

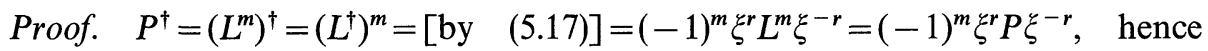
$\left(P \xi^{-r}\right)^{\dagger}=(-1)^{r} \xi^{-r} P^{\dagger}=(-1)^{m+r} P \xi^{-r}$. Using $P=P_{\geqq r}+P_{<r}$, we rewrite (5.18)in the form

$$
\left(P_{\geqq r} \xi^{-r}\right)^{\dagger}+\left(P_{<r} \xi^{-r}\right)^{\dagger}=(-1)^{m+r}\left[P_{\geqq r} \xi^{-r}+P_{<r} \xi^{-r}\right],
$$

and picking out the differential part of (5.20) yields (5.19).

We have to show that the relations $L^{\dagger}=-\xi^{k} L \xi^{-k}$ and $L_{t}=\left[P_{\geqq k}, L\right]$ result in

$$
L_{t}^{\dagger}=-\xi^{k} L_{t} \xi^{-k} \text {. }
$$


The left-hand side of (5.21) is $\left(\left[P_{\geq k}, L\right]\right)^{\dagger}=\left[L^{\dagger},\left(P_{\geq k}\right)^{\dagger}\right]=[$ by (5.19) with $r=k]$ $=\left[-\xi^{k} L \xi^{-k},-\xi^{k} P_{\geqq k} \xi^{-k}\right]=\xi^{k}\left[L, P_{\geqq k}\right] \xi^{-k}=-\xi^{k}\left[P_{\geqq k}, L\right] \xi^{-k}$, which is the right-hand side of 5.21 .

Remark 5.22. The KdV hierarchy is associated with the Lax operator $L=\xi^{2}+h$. This operator can be viewed as a member of two different series: 1) general scalar Lax operators $\left.L=\sum_{i=0}^{n} u_{i} \xi^{i}, u_{n}=1, u_{n-1}=0 ; 2\right)$ specialized Lax operators $L=\sum_{i=0}^{n} u_{i} \xi^{i}, u_{n}=1, u_{n-1}^{i=0}=0$, satisfying the condition $L^{\dagger}=(-1)^{n} L$. The second point of view, which we took in Theorem 5.10, leads to a rather nontrivial theory in the standard case $k=0([19,20])$.

Remark 5.23. The flows (5.12)-(5.14) are all nontrivial except when $k=0$ and $m \in \mathbb{N} n$, which can be seen by going to the quasiclassical limit and changing the commutator in the right-hand side (5.12) into the Poisson bracket. The same type of reasoning shows that the c.l.'s $H_{2 M+1}$ remain nontrivial after specialization (5.13) is imposed. It seems very likely that the c.l's $H_{2 M}$ become trivial but I couldn't prove this in general.

We conclude this section by discussing other specializations of the general operator

$$
L=\sum_{-k}^{n} u_{i} \xi^{i}
$$

for $k=1$, 2. Firstly, since

$$
L_{t}=\left[\left(\left(P^{\dagger}\right)_{\geqq k}\right)^{\dagger}, L\right],
$$

it follows that $\partial_{t}\left(L_{<0}\right)=\left[\left(\left(P^{\dagger}\right)_{\geqq k}\right)^{\dagger}, L_{<0}\right]_{<0}$, and hence

$$
\left\{u_{i}=0 \mid-k \leqq i<0\right\}
$$

is an invariant submanifold (speaking geometrically) of (5.25). Now assume that we have already reduced (5.24) on (5.26) and let us write the resulting $L$ in the left form:

$$
L=\sum_{i=0}^{n} \xi^{i} v_{i}
$$

If $P=\sum_{s} \xi^{s} p_{s}$, then

$$
L_{t}=\left[\sum_{s \geqq k} \xi^{s} p_{s}, \sum_{i \geqq 0} \xi^{i} v_{i}\right] .
$$

It follows that (5.28) has the invariant submanifold $J_{r}^{k}:=\left\{v_{i}=0 \mid 0 \leqq i \leqq r\right\}$ for each $r<k$. Thus, we have three possibilities:

$$
\begin{gathered}
J_{0}^{1}=\left\{v_{0}=0\right\}, k=1, \\
J_{0}^{2}=\left\{v_{0}=0\right\}, k=2, \\
J_{1}^{2}=\left\{v_{0}=v_{1}=0\right\}, k=2 .
\end{gathered}
$$

Consider for example the first nontrivial case: $n=2$ on $J_{0}^{1}$ (5.29). Here

$$
L=\xi^{2}+\xi v,
$$


and for $P=\xi$ we obtain $v_{t}=\partial v$. The next nontrivial flow is provided by $P=L^{3 / 2}$ : since $L^{1 / 2}=\xi+\frac{1}{2} v+a \xi^{-1}+b \xi^{-2}+\ldots$, where $a=\frac{1}{4} v_{x}-\frac{1}{8} v^{2}, b=-\frac{1}{2}(\partial+v)(a)$, we have $\left(\left(\left(L^{3 / 2}\right)^{\dagger}\right)_{\leqq 0}\right)^{\dagger}=-b+\ldots$. Hence $L_{t}=\xi v_{t}=\left[\xi^{2}+\xi v,-b+\ldots\right]=\xi 2 \partial(-b)$ $=\xi \partial(\partial+v)(a)$, so that

$$
v_{t}=\partial\left(v_{x x} / 4-v^{3} / 8\right)=\left(2 v_{x x x}-3 v^{2} v_{x}\right) / 8,
$$

which is just the modified $\mathrm{KdV}(\mathrm{m}-\mathrm{KdV})$ equation.

Remark 5.34. The Lax representation $\left(\xi^{2}+\xi v\right)_{t}=\left[K, \xi^{2}+\xi v\right]$ with $K=\xi^{3}$ $+\frac{3}{2} v \xi^{2}+\left(\frac{3}{4} v^{2}+\frac{9}{4} v_{x}\right) \xi+\frac{3}{4}\left(v^{2}+v_{x}\right)_{x}\left[=\xi^{3}+\frac{3}{2} \xi^{2} v+\xi \frac{3}{4}\left(v^{2}-v_{x}\right)=\left(\left(\left(L^{3 / 2}\right)^{\dagger}\right)_{\geqq 1}\right)^{\dagger}\right]$ for the $\mathrm{m}-\mathrm{KdV}$ Eq. (5.33) was found by Knörrer [21].

Let us show that the higher flows

$$
L_{t}=\left[\left(\left(P^{\dagger}\right)_{\geqq 1}\right)^{\dagger}, L\right], L=\xi^{2}+\xi v, P=L^{m / 2}, m \equiv 1(\bmod 2)
$$

are exactly the higher $\mathrm{m}-\mathrm{KdV}$ equations (for even $m, P=\left(\left(P^{\dagger}\right)_{\geqq 1}\right)^{\dagger}$ and $L_{t}=\left[\left(\left(P^{\dagger}\right)_{\geqq 1}\right)^{\dagger}, L\right]$ is a trivial equation). First, the quasiclassical arguments show that Eqs. (5.35) are all nontrivial, and c.l.'s $H_{2 m+1}=\frac{2}{2 m+1} \operatorname{Res} L^{(2 m+1) / 2}$ are nontrivial as well. They are also homogeneous (of weight $2 m+2$ ) in the grading $r k v^{(j)}=j+1$. Now, for $L^{m / 2}=\sum \xi^{s} p_{s}(m)$ we have

$$
L_{t}=\xi v_{t}=\left[-\sum_{s \leqq 0} \xi^{s} p_{s}(2 m+1), \xi^{2}+\xi v\right] \geqq 1=\xi 2 \partial p_{0}(2 m+1),
$$

so that

$$
v_{t}=2 \partial p_{0}(2 m+1)
$$

Also, $\quad$ since $\quad d H_{m}=\frac{2}{m} d \operatorname{Res} L^{m / 2} \sim \operatorname{Res}\left(d L \circ L^{(m-2) / 2}\right)=\operatorname{Res}\left[\xi d v \sum \xi^{s} p_{s}(m-2)\right]$ $\sim \operatorname{Res}\left[d v \sum \xi^{s} p_{s}(m-2) \xi\right]=d v\left[p_{-2}(m-2)-\partial p_{-1}(m-2)\right]$ by (1.16), we obtain

$$
p_{-2}(m)-\partial p_{-1}(m)=\frac{\delta H_{m+2}}{\delta v} .
$$

It's not exactly what we need, which is $p_{0}(m)$, as (5.36) shows. We continue as follows. From the double identity

$$
\sum \xi^{s} p_{s}(m+2)=\left(\xi^{2}+\xi v\right) \sum \xi^{s} p_{s}(m)=\sum \xi^{s} p_{s}(m)\left(\xi^{2}+\xi v\right),
$$

we find

$$
\begin{gathered}
p_{-1}(m+2)=p_{-3}(m)+v p_{-2}(m)+v^{(1)} p_{-1}(m) \\
=p_{-3}(m)-2 \partial p_{-2}(m)+\partial^{2} p_{-1}(m)+p_{-2}(m) v-v \partial p_{-1}(m), \\
p_{0}(m+2)=p_{-2}(m)+v p_{-1}(m) .
\end{gathered}
$$

Comparing (5.39) and (5.40) we find

$$
v p_{-1}(m)=-2 p_{-2}(m)+\partial p_{-1}(m)
$$

and substituting this into (5.41) we obtain

$$
p_{0}(m+2)=-p_{-2}(m)+\partial p_{-1}(m) .
$$


Together with (5.37) this results in

$$
p_{0}(m)=-\frac{\delta H_{m}}{\delta v}
$$

and (5.36) finally yields

$$
v_{t}=-\partial \frac{\delta H_{2 m+1}}{\delta v}
$$

which is the standard Hamiltonian form of the $\mathrm{m}-\mathrm{KdV}$ hierarchy. Since $r k H_{2 m+1}=2 m+2$ and $H_{2 m+1} \nsim 0$, the uniqueness of the conservation laws of the m-KdV equation [22] implies that $H_{2 m+1}$, up to a constant multiple, is equivalent to the standard c. 1 . \# $(2 m+1)$ of the $\mathrm{m}-\mathrm{KdV}$ hierarchy. Since the Hamiltonian form of this hierarchy is the same as (5.45), the flows too differ at most by a constant multiple.

In most theories of integrable systems the centerpiece is a (generalized) Miura map. Since we have realized the classical modified $\mathrm{KdV}$ hierarchy inside the $k=1$ case of the general system (2.3), the reader will undoubtedly wonder about the realization of the classical Miura map in our general context. Here is the answer. For $k=1$ and $L=\sum^{n} \xi^{i} u_{i}, P=L^{m / n}=\sum_{s} p_{s} \xi^{s}$, with $L_{t}=\left[P_{\geqq 1}, L\right]=\left[-P_{\leqq 0}, L\right]$, we have $u_{n-1, t}=n \partial p_{0}$. Therefore, if we introduce a new variable $w: \partial w=-\frac{1}{n} u_{n-1}$, we can lift the system $L_{t}=\left[P_{\geqq 1}, L\right]$ from the ring $C_{1}=C_{u_{n-1}, u_{n-2}, \ldots}$ into the ring $C_{2}=C_{w, u_{n-2}, \ldots .}$. Now consider the following conjugation:

$$
\mathscr{L}=e^{-w} L e^{w}, \mathscr{P}=e^{-w} P e^{w}=\mathscr{L}^{m / n} .
$$

Then

$$
\begin{aligned}
\mathscr{L}_{t} & =e^{-w} L_{t} e^{w}+\left[\mathscr{L}, w_{t}\right]=\left[\mathscr{L}, e^{-w} P_{\leqq 0} e^{-w}-p_{0}\right] \\
& =\left[\mathscr{L},-e^{-w} P_{\geqq 1} e^{w}-p_{0}\right]=\left[\mathscr{L}, \mathscr{F}_{<0}\right]=\left[\mathscr{L},-\mathscr{H}_{\geqq 0}\right],
\end{aligned}
$$

where $\mathscr{F}=\mathscr{F}_{<0}=e^{-w} P_{\leqq 0} e^{w}-p_{0}, \mathscr{H}=\mathscr{H}_{\geqq 0}=e^{-w} P_{\geqq 1} e^{w}+p_{0}$. Since $\mathscr{F}+\mathscr{H}=\mathscr{P}$, we see that $\mathscr{F}=\mathscr{P}_{<0}, \mathscr{\mathscr { H }}=\mathscr{P}_{\geqq 0}$, and we arrive at the standard $k=0$-case

$$
\mathscr{L}_{t}=\left[\mathscr{P}_{\geqq 0}, \mathscr{L}\right]=\left[-\mathscr{P}_{<0}, \mathscr{L}\right] \text {. }
$$

The map $L \rightarrow \mathscr{L}=e^{-w} L e^{w}$ is the desired Miura map. Since $e^{-w} \xi^{i} e^{w}=\left(\xi+w^{(1)}\right)^{i}=\left(\xi-\frac{1}{n} u_{n-1}\right)^{i}$, we see that the Miura map amounts to changing $\xi$ into $\xi-\frac{1}{n} u_{n-1}$ :

$$
\sum^{n} \xi^{j} U_{j}=\sum^{n}\left(\xi-\frac{1}{n} u_{n-1}\right)^{i} u_{i}, U_{n}=1, U_{n-1}=0, u_{n}=1 \text {. }
$$

In particular, for $L=\xi^{2}+\xi v(5.32), \xi^{2}+u=\left(\xi-\frac{1}{2} v\right)^{2}+\left(\xi-\frac{1}{2} v\right) v=\xi^{2}+\frac{1}{2} v_{x}-\frac{1}{4} v^{2}$, hence $u=\frac{v_{x}}{2}-\frac{v^{2}}{4}$, and this is the usual Miura map. For $n=1$, we have from (5.48): 


$$
\begin{aligned}
\sum_{-\infty}^{1}\left(\xi-u_{0}\right)^{i} u_{i} & =\xi-u_{0}+u_{0}+\sum_{i \geqq 0}\left(\xi-u_{0}\right)^{-i-1} u_{-i-1} \\
& =[\text { by the adjoint of }(4.9)] \\
& =\xi+\sum_{i \geqq 0} \sum_{\alpha \geqq 0} \xi^{-i-1-\alpha}(-1)^{\alpha}\left(\begin{array}{c}
-i-1 \\
\alpha
\end{array}\right) Q_{\alpha}\left(u_{0}\right) u_{-i-1},
\end{aligned}
$$

so that

$$
U_{-k-1}=\sum_{i+\alpha=k}(-1)^{\alpha}\left(\begin{array}{c}
-i-1 \\
\alpha
\end{array}\right) Q_{\alpha}\left(u_{0}\right) u_{-i-1} .
$$

Suppose now, that $L=\xi+u+\xi^{-1} h$. Then

$$
\mathscr{L}=\xi+(\xi-u)^{-1} h=[\text { by }(4.16)]=\xi+\sum_{i \geqq 0} \xi^{-i-1} Q_{i}(u) h=\xi+\sum_{i \geqq 0} \xi^{-i-1} A_{i},
$$

and Theorem 4.4 (for $r=0$ ) says that the Miura map (5.50) is canonical, as it should be in general. It is very likely that the full Miura map (5.49) is also canonical between the Hamiltonian structures $B^{0}(2.17)$ of the $k=0$-case and (2.20) of the $k=1$-case respectively; the quasi-classical limit of this map is indeed canonical between the quasiclassical limits of the corresponding Hamiltonian structures (this is another generalization, different from the $r=0$-case of Theorem 4.4 , of the compatibility Theorem 0.7 in [5]).

Remark 5.48. In physical language, the interpretation of the Miura map as a conjugation was proposed by J. Gibbons [23].

Acknowledgements. During my visit to Tuscon in August 1982, I had useful discussions with H. Flaschka and A. Newell about the Broer-Kaup system. This work was partially supported by the NSF and DOE.

\section{References}

1. Broer, L.J.F.: Approximate equations for long water waves. Appl. Sci. Res. 31, 377-395 (1975)

2. Kaup, D.J.: A higher-order water-wave equation and the method for solving it. Prog. Theor. Phys. 54, 396-408 (1975)

3. Matveev, V.B., Yavor, M.I.: Solutions presque périodiques et a $N$-solitons de l'équation hydrodynamique non linéaire de Kaup. Ann. Inst. H. Poincare, Sect. A, XXXI, No. 1, 25-41 (1979)

4. Wilson, G.: Commuting flows and conservation laws for Lax equations. Math. Proc. Cambr. Phil. Soc. 86, 131-143 (1979)

5. Manin, Yu.I.: Algebraic aspects of non-linear differential equations. Itogi Nauki Tekh., Ser. Sovr. Prob. Math. 11, 5-152 (1978) [J. Sov. Math. 11, 1-122 (1979)]

6. Kupershmidt, B.A.: Discrete Lax equations and differential-difference calculus. E.N.S. Lecture Notes, Paris (1982); Revue Asterisque, Vol. 123, Paris (1985)

7. Gel'fand, I.M., Dorfman, I.Ya.: Hamiltonian operators and infinite-dimensional Lie algebras. Funct. Anal. Appl. 15, 23-40 (1981) (Russian); 173-187 (English)

8. Kupershmidt, B.A.: On dual spaces of differential Lie algebras. Physica 7D, 334-337 (1983)

9. Kupershmidt, B.A.: On algebraic models of dynamical systems. Lett. Math. Phys. 6, 85-89 (1982) 
10. Kupershmidt, B.A.: Normal and universal forms in integrable hydrodynamical systems. In Proc. NASA Ames-Berkeley 1983 conf. on nonlinear problems in optimal control and hydrodynamics. Hunt, R.L., Martin, C., eds. Math. Sci. Press (1984)

11. Benney, D.J.: Some properties of long nonlinear waves. Stud. Appl. Math. L11 (1), 45-50 (1973)

12. Kupershmidt, B.A., Manin, Yu.I.: Long-wave equation with free boundaries. I. Conservation Laws. Funct. Anal. Appl. 11: 3, 31-42 (1977) (Russian); 188-197 (English)

13. Kupershmidt, B.A., Manin, Yu.I.: Equations of long waves with a free surface. II. Hamiltonian structure and higher equations. Funct. Anal. Appl. 12: 1, 25-37 (1978) (Russian); 20-29 (English)

14. Kupershmidt, B.A.: Modern Hamiltonian formalism (to appear)

15. Kupershmidt, B.A., Wilson, G.: Modifying Lax equations and the second Hamiltonian structure. Invent. Math. 62, 403-436 (1981)

16. Kupershmidt, B.A.: Deformations of Hamiltonian structures. M.I.T. preprint (1978)

17. Kupershmidt, B.A., Wilson, G.: Conservation laws and symmetries of generalized SineGordon equations. Commun. Math. Phys. 81, 189-202 (1981)

18. Wilson, G.: The modified Lax and two-dimensional Toda lattice equations associated with simple Lie algebras. Ergod. Th. Dynam. Syst. 1, 361-380 (1981)

19. Drinfel'd, V.G., Sokolov, V.V.: Equations of KdV type and simple Lie algebras. Dokl. Akad. Nauk SSSR 258, 11-16 (1981) [Sov. Math. Dokl. 23, 457-462 (1981)]

20. Drinfel'd, V.G., Sokolov, V.V.: Lie algebras and the Korteweg-de-Vries type equations. Itogi Nauki Tekh., Ser. Sovr. Prob. Math., Vol. 24. Moscow (1984)

21. Knörrer, H.: Private communication, Bonn (June, 1981)

22. Kupershmidt, B.A.: Involutivity of conservation laws for a fluid of finite depth and BenjaminOno equations. Libertas Math. 1, 189-202 (1981)

23. Gibbons, J.: Related integrable hierarchies. I. Two nonlinear Schrödinger equations. DIAS preprint STP-81-06 (Dublin, 1981)

Communicated by L. Nirenberg

Received May 18, 1984 
